\title{
Effects of budesonide combined with noninvasive ventilation on PCT, STREM-1, chest lung compliance, humoral immune function and quality of life in patients with AECOPD complicated with type II respiratory failure
}

https://doi.org/10.1515/med-2019-0023

received May 15, 2018; accepted November 20, 2018

\begin{abstract}
Objective: Our objective is to explore the effects of budesonide combined with noninvasive ventilation on procalcitonin (PCT), soluble myeloid cell triggering receptor-1 (sTREM-1), thoracic and lung compliance, humoral immune function, and quality of life in patients with acute exacerbation of chronic obstructive pulmonary disease (AECOPD) complicated with type II respiratory failure. Methods: There were 82 patients with AECOPD complicated with type II respiratory failure admitted into our hospital between March, 2016-September, 2017. They were selected and randomly divided into observation group $(n=41)$ and control group ( $n=41)$. The patients in the control group received noninvasive mechanical ventilation and the patients in the observation group received budesonide based on the control group. The treatment courses were both 10 days. Results: The total effective rate in the observation group (90.25\%) was higher than the control group $(65.85 \%)(\mathrm{P}<0.05)$. The scores of cough, expectoration, and dyspnea were decreased after treatment (Observation group: $\mathrm{t}=18.7498,23.2195,26.0043$, control group: $\mathrm{t}=19.9456,11.6261,14.2881, \mathrm{P}<0.05)$; the scores of cough, expectoration, and dyspnea in the observation group were lower than the control group after treatment $(\mathrm{t}=11.6205,17.4139,11.6484, \mathrm{P}<0.05)$. $\mathrm{PaO} 2$
\end{abstract}

\footnotetext{
*Corresponding author: Jianping Wang, Department of Emergency, 1st Hospital of Yulin, Yulin City, Shaanxi Province 719000, China; Email: gaoerxiangabc@163.com

Erxiang Gao, Emergency Department, Xingyuan Hospital of Yulin, Shaanxi 719000, China
}

Chi Zhang, The Second Department of Internal Medicine, Xingyuan Hospital of Yulin, Shaanxi 719000, China

\#These authors contributed equally to this work. was increased and $\mathrm{PaCO} 2$ was decreased in both groups after treatment (Observation group: $\mathrm{t}=24.1385,20.7360$, control group: $\mathrm{t}=11.6606,9.2268, \mathrm{P}<0.05) ; \mathrm{PaO} 2$ was higher and $\mathrm{PaCO} 2$ was lower in the observation group than the control group after treatment $(\mathrm{t}=10.3209,12.0115, \mathrm{P}<0.05)$. Serum PCT and sTREM-1 in both groups were decreased after treatment (Observation group: $\mathrm{t}=16.2174,12.6698$, control group: $\mathrm{t}=7.2283,6.1634, \mathrm{P}<0.05)$; serum PCT and STREM-1 in the observation group were lower than the control group after treatment $(\mathrm{t}=10.1017,7.8227, \mathrm{P}<0.05)$. The thoracic and lung compliance in both groups were increased after treatment (Observation group: $t=30.5359$, 17.8471, control group: $\mathrm{t}=21.2426,13.0007, \mathrm{P}<0.05)$; the thoracic and lung compliance in the observation group were higher than the control group after treatment $(\mathrm{t}=10.8079$, 5.9464, $\mathrm{P}<0.05)$. IgA and $\operatorname{IgG}$ in both groups were increased after treatment (Observation group: $t=9.5794$, 25.3274, control group: $\mathrm{t}=5.5000,4.7943, \mathrm{P}<0.05$ ), however IgM was not statistically different after treatment (Observation group: $\mathrm{t}=0.7845$, control group: $\mathrm{t}=0.1767, \mathrm{P}>0.05$ ); IgA and IgG in the observation group were higher than the control group $(t=4.9190,4.7943, \mathrm{P}<0.05)$, however IgM was not statistically different between two groups after treatment $(\mathrm{t}=0.6168, \mathrm{P}>0.05)$. COPD assessment test (CAT) scores were decreased in both groups after treatment (Observation group: $t=20.6781$, control group: $t=9.0235$, $\mathrm{P}<0.05$ ); CAT score in the observation group was lower than the control group after treatment $(\mathrm{t}=12.9515, \mathrm{P}<0.05)$. Forced expiratory volume in one second (FEV1\%) and forced expiratory volume in one second/ forced expiratory volume in one second (FEV1/FVC) were increased in both groups after treatment (Observation group: $\mathrm{t}=15.3684$, 15.9404, control group: $\mathrm{t}=10.6640,12.8979, \mathrm{P}<0.05)$; FEV1\% and FEV1/FVC in the observation group were higher than the control group $(\mathrm{t}=6.9528,7.3527, \mathrm{P}<0.05)$. The rates of complication were not statistically different between 
two groups ( $\mathrm{P}>0.05)$. Conclusion: Budesonide combined with noninvasive mechanical ventilation has good curative effects in treating AECOPE patients complicated with type II respiratory failure. It can decrease serum PCT and sTREM-1, increase thoracic lung compliance, and improve the humoral immune function and life quality.

Keywords: Budesonide; Noninvasive ventilation; Acute exacerbation of chronic obstructive pulmonary disease; Type II respiratory failure; Procalcitonin; Soluble myeloid cell triggering receptor-1; Thoracic and lung compliance; Humoral immune function; Quality of life

\section{Introduction}

Chronic obstructive pulmonary disease (COPD) is a common respiratory disease with an increasing incidence rate that has significantly affected the life quality of people [1-3]. Acute exacerbation of chronic obstructive pulmonary disease (AECOPD) can cause complications such as respiratory failure that endangers patients' lives [4-6]. Thus, an effective curative method is especially important. Noninvasive positive pressure ventilation (noninvasive mechanical ventilation) is the primary method to treat AECOPD complicated with respiratory failure [5,7-9]. In the recent years, it is reported that aerosol inhalation of medicine can directly act on the airway, which in return has significant effect on AECOPD complicated with respiratory failure [9-12]. Thus, in this study, we analyzed the effects of budesonide combined with noninvasive ventilation on procalcitonin (PCT), soluble myeloid cell triggering receptor-1 (sTREM-1), thoracic and lung compliance, and humoral immune function and quality of life in patients with AECOPD complicated with type II respiratory failure.

\section{Materials and methods}

\subsection{General data}

Eighty-two patients with AECOPD complicated with type II respiratory failure admitted into our hospital between March, 2016-September, 2017 were selected. The criteria was based on the diagnostic criteria of COPD [13]. The patients were divided into observation group $(n=41)$ and control group $(n=41)$. In the observation group, there were 15 female patients and 26 male patients. The age of patients was 50-78 years old and the average age was (65.98 \pm 4.87$)$ years old; in the control group, there were 17 female patients and 24 male patients. The age of patients was 51-80 years old and the average age was $(66.71 \pm 5.13)$ years old. The general data were comparable between two groups.

\subsection{Inclusion and exclusion criteria}

\subsubsection{Inclusion criteria}

The patients met the criteria of AECOPD complicated with type II respiratory failure, and were also confirmed with past history, physical signs, blood routine test and X-Ray, and a severe AECOPD stage; the age of patients was 50-80 years.

Ethics approval and consent to participate: This study was approved by the Committee on Ethics of Biomedicine of Yulin First People's Hospital. This study also complied with the Declaration of Helsinki, and signed, written informed consent was obtained from all subjects included in this study.

\subsubsection{Exclusion criteria}

The patients complicated with severe electrolyte disturbance, acute cerebrovascular disease, or severe cerebral vessel sclerosis; the patients complicated with severe liver or kidney dysfunction; the patients had lots of secretion in the airway, which required emergent tracheal intubation; the patients had mental disorder.

\subsection{Curative method}

The patients in the two groups received routine treatment, including oxygen inhalation, bronchodilator, anti-infection and expectorant. Control group: noninvasive mechanical ventilation was applied as the following: a proper size nasal/face mask was selected and fixed with headband, the mask was connected to the ventilator, and then the ventilator parameters were set as the following: the inspiration pressure was initially $8-10 \mathrm{cmH}_{2} \mathrm{O}$ and gradually increased to $12-20 \mathrm{cmH}_{2} \mathrm{O}$ which fitted the tolerance of patients; the respiratory rate was $12-20 / \mathrm{min}$; the oxygen flow rate was 4-6 L/min, the positive end-expiratory pressure was 3-5 $\mathrm{cmH}_{2} \mathrm{O}$, most of patients received intermittent ventilation, and the duration was $\geq 4 \mathrm{~h} /$ time 
and $\geq 12 \mathrm{~h}$ /day. Observation group: the patients received series connection of budesonide suspension with ventilator circuit (AstraZeneca Pty Ltd., Sydney, Australia), 2 mg/ time, 3 times/day. The treatment courses in both groups were 7 days.

\subsection{Criteria for curative efficacy}

Significant improvement: The major symptoms including cough, expectoration, and dyspnea almost disappeared, rale was significantly improved, chest imaging showed that the lung markings and shadow were significantly improved; improvement: The major symptoms including cough, expectoration and dyspnea were improved, rale was improved, chest imaging showed that the lung markings and shadow were improved; invalidity: The major symptoms including cough, expectoration, dyspnea, rale, and chest imaging were not improved. The total effective rate $=($ case number of significant improvement + case number of improvement)/total case number $\times 100 \%$.

\subsection{Observational indexes}

The major symptoms (cough, expectoration, and dyspnea) before and after treatment in two groups were observed and evaluated as none ( 0 point), mild (1 point), moderate ( 2 points), and severe ( 3 points) based on the score; the changes of blood gas parameters before and after treatment in the two groups were observed; the changes of PCT and STREM- 1 before and after treatment in the two groups were observed: first $3 \mathrm{ml}$ peripheral venous blood was collected before and after treatment and the serum was separated by centrifugation at $15 \mathrm{~cm}$ diameter and $2500 \mathrm{r} / \mathrm{min}$ for 24 hours; the changes of thoracic and lung compliance before and after treatment in the two groups were observed; the changes of humoral immune function before and after treatment in the two groups were observed, including IgA, IgG and IgM in above serum sample detected by Hitachi 7600 modular chemistry analyzer (Hitachi, Ltd.,
Tokyo, Japan); the improvement of life quality before and after treatment in the two groups were observed, COPD assessment test (CAT) was applied including 6 subjective indexes of tight chest, cough, energy, phlegm, emotion and sleep and 2 tolerance indexes including exercise tolerance and daily exercise influence, 5 points per item, and the total CAT scores range from 0-40. Higher scores denote a more severe impact of COPD on a patient's life; pulmonary functions in both groups before and after treatment were observed, including forced expiratory volume in one second (FEV1), forced vital capacity (FVC) and a percentage of the forced vital capacity (FEV1\%); the complications in two groups were observed.

\subsection{Statistical analysis.}

The enumeration data and measurement data were analyzed by SPSS13.0. All the statistical significance was presented as $P<0.05$. The enumeration data were analyzed by $x^{2}$ and presented as percentage; the measurement data were analyzed by $t$ test and presented as mean \pm SD.

\section{Results}

\subsection{Comparison of total effective rate between two groups}

As shown in Table 1, the total effective rate in the observation group $(90.25 \%)$ was higher than control group $(65.85 \%)(P<0.05)$

\subsection{Comparison of major symptoms between two groups}

As shown in Table 2, the scores of cough, expectoration, and dyspnea were not statistically different between two groups before treatment; the score of cough, expectora-

Table 1: Comparison of total effective rate between two groups

\begin{tabular}{llllll}
\hline Group & Case number & $\begin{array}{l}\text { Significant } \\
\text { improvement (\%) }\end{array}$ & Improvement (\%) & Invalidity (\%) & $\begin{array}{l}\text { Total effective rate } \\
\text { (\%) }\end{array}$ \\
\hline Observation group & 41 & $23(56.10)$ & $14(34.15)$ & $4(9.75)$ & $37(90.25)$ \\
Control group & 41 & $14(34.15)$ & $13(31.70)$ & $14(34.15)$ & $27(65.85)$ \\
x2 & - & - & - & - & 7.1181 \\
P & - & - & - & - & $<0.05$ \\
\hline
\end{tabular}


tion, and dyspnea were decreased after treatment (Observation group: $t=18.7498,23.2195,26.0043$, control group: $t=19.9456,11.6261,14.2881, P<0.05)$; the scores of cough, expectoration, and dyspnea in the observation group were lower than the control group after treatment $(t=11.6205$, 17.4139, 11.6484, $P<0.05)$.

\subsection{Comparison of blood gas parame- ters between two groups before and after treatment}

As shown in Table 3, $\mathrm{PaO}_{2}$ and $\mathrm{PaCO}_{2}$ were not statistically different between two groups before treatment $(t=1.1743,0.4931, P>0.05) ; \mathrm{PaO}_{2}$ was increased and $\mathrm{PaCO}_{2}$ was decreased in both groups after treatment (Observation group: $t=24.1385,20.7360$, control group: $t=11.6606$,
9.2268, $P<0.05)$; $\mathrm{PaO}_{2}$ was higher and $\mathrm{PaCO}_{2}$ was lower in the observation group than the control group after treatment $(t=10.3209,12.0115, P<0.05)$.

\subsection{Comparison of serum PCT and STREM-1 between two groups before and after treatment}

As shown in Table 4, serum PCT and sTREM-1 were not statistically different between two groups before treatment $(t=0.4159,0.6173, P>0.05)$; Serum PCT and sTREM- 1 in both groups were decreased after treatment (observation group: $t=16.2174,12.6698$, control group: $t=7.2283,6.1634$, $P<0.05)$; serum PCT and STREM-1 in the observation group were lower than the control group after treatment $(t=10.1017,7.8227, P<0.05)$.

Table 2: Comparison of major symptoms between two groups ( $\left.\bar{x} \bar{X}_{ \pm \mathrm{s}}\right)$

\begin{tabular}{llllll}
\hline Group & & Case number & Cough (point) & Expectoration (point) & Dyspnea (point) \\
\hline Observation group & Before treatment & 41 & $1.94 \pm 0.45$ & $1.87 \pm 0.39$ & $1.82 \pm 0.31$ \\
& After treatment & 41 & $0.56 \pm 0.14 \mathrm{ab}$ & $0.41 \pm 0.10 \mathrm{ab}$ & $0.47 \pm 0.12 \mathrm{ab}$ \\
\multirow{2}{*}{ Control group } & Before treatment & 41 & $1.96 \pm 0.40$ & $1.85 \pm 0.45$ & $1.84 \pm 0.36$ \\
& After treatment & 41 & $1.08 \pm 0.25 \mathrm{a}$ & $0.97 \pm 0.18 \mathrm{a}$ & $0.91 \pm 0.21 \mathrm{a}$ \\
\hline
\end{tabular}

Note: ${ }^{\mathrm{a}}<0.05$ compared with before treatment in the same group; ${ }^{\mathrm{b}} P<0.05$ compared with the control group after treatment.

Table 3: Comparison of blood gas parameters between two groups before and after treatment $\left(\bar{x} \bar{x}_{ \pm \mathrm{s}}\right)$

\begin{tabular}{lllll}
\hline Group & & Case number & $\mathrm{PaO}_{2}(\mathrm{mmHg})$ & $\mathrm{PaCO}_{2}(\mathrm{mmHg})$ \\
\hline Observation group & Before treatment & 41 & $52.18 \pm 2.45$ & $65.98 \pm 3.25$ \\
& After treatment & 41 & $70.38 \pm 4.16^{\mathrm{ab}}$ & $52.13 \pm 2.78^{\mathrm{ab}}$ \\
\multirow{2}{*}{ Control group } & Before treatment & 41 & $52.85 \pm 2.71$ & $66.34 \pm 3.36$ \\
& After treatment & 41 & $61.32 \pm 3.78^{\mathrm{a}}$ & $59.83 \pm 3.02^{\mathrm{a}}$ \\
\hline
\end{tabular}

Note: ${ }^{a}<<0.05$ compared with before treatment in the same group; ${ }^{b} P<0.05$ compared with the control group after treatment.

Table 4: Comparison of serum PCT and STREM-1 between two groups before and after treatment $\left(\bar{x} \bar{X}_{ \pm s}\right)$

\begin{tabular}{lllll}
\hline Group & & Case number & PCT (ng/L) & STREM-1(ng/L) \\
\hline Observation group & Before treatment & 41 & $613.25 \pm 24.25$ & $94.52 \pm 3.67$ \\
& After treatment & 41 & $536.29 \pm 18.31^{\mathrm{ab}}$ & $85.41 \pm 2.78^{\mathrm{ab}}$ \\
\multirow{2}{*}{ Control group } & Before treatment & 41 & $615.46 \pm 23.87$ & $95.03 \pm 3.81$ \\
& After treatment & 41 & $579.83 \pm 20.65^{\mathrm{a}}$ & $90.38 \pm 2.97^{\mathrm{a}}$ \\
\hline
\end{tabular}

Note: ${ }^{a}<<0.05$ compared with before treatment in the same group; ${ }^{b} P<0.05$ compared with the control group after treatment. 


\subsection{Comparison of humoral immune function between two groups}

As shown in Table 5, IgA, IgG and IgM were not statistically different between the two groups before treatment ( $t=0.5486,0.1484,0.3412, P>0.05)$; IgA and IgG in both groups were increased after treatment (Observation group: $t=9.5794,25.3274$, control group: $t=5.5000$, 4.7943, $P<0.05$ ), however IgM was not statistically different in both groups after treatment (observation group: $t=0.7845$, control group: $t=0.1767, P>0.05$ ); IgA and IgG in the observation group were higher than the control group $(t=4.9190$, 4.7943, $P<0.05$ ), however IgM was not statistically different between two groups after treatment $(t=0.6168, P>0.05)$.

\subsection{Comparison of thoracic and lung com- pliance between two groups before and after treatment}

As shown in Table 6, thoracic and lung compliance were not statistically different between the two groups before treatment $(t=0.5551,0.5778, P>0.05)$; The thoracic and lung compliance in both groups were increased after treatment. (Observation group: $t=30.5359,17.8471$, control group: $t=21.2426,13.0007, P<0.05)$; The thoracic and lung compliance in the observation group were higher than the control group after treatment $(t=10.8079,5.9464, P<0.05)$.

\subsection{Comparison of CAT score between two groups before and after treatment}

As shown in Table 7, CAT scores were not statistically different between the two groups before treatment $(t=0.3993, P>0.05)$; CAT scores were decreased in both groups after treatment (observation group: $t=20.6781$, control group: $t=9.0235, P<0.05)$; CAT score in the observation group was lower than the control group after treatment $(t=12.9515, P<0.05)$.

\subsection{Comparison of pulmonary functions between two groups before and after treatment}

As shown in Table 8, FEV1\% and FEV1/FVC were not statistically different between the two groups before treatment $(t=0.8258,1.2307, P>0.05):$ FEV1 $\%$ and FEV1/FVC were increased in both groups after treatment (Observation group: $t=15.3684,15.9404$, control group: $t=10.6640$, 12.8979, $P<0.05$ ); FEV1\% and FEV1/FVC in the observation group were higher than the control group $(t=6.9528$, $7.3527, P<0.05)$.

Table 5: Comparison of humoral immune function between two groups $\left(\bar{X} \bar{X}_{ \pm S}\right)$

\begin{tabular}{llllll}
\hline Group & & Case number & IgA (g/L) & IgG (g/L) & IgM (g/L) \\
\hline Observation group & Before treatment & 41 & $1.94 \pm 0.17$ & $10.12 \pm 1.21$ & $1.36 \pm 0.25$ \\
& After treatment & 41 & $2.38 \pm 0.24^{\mathrm{ab}}$ & $12.89 \pm 1.37^{\mathrm{ab}}$ & $1.40 \pm 0.21$ \\
\multirow{2}{*}{ Control group } & Before treatment & 41 & $1.92 \pm 0.16$ & $10.08 \pm 1.23$ & $1.38 \pm 0.28$ \\
& After treatment & 41 & $2.14 \pm 0.20^{\mathrm{a}}$ & $11.42 \pm 1.30^{\mathrm{a}}$ & $1.37 \pm 0.23$ \\
\hline
\end{tabular}

Note: ${ }^{a} P<0.05$ compared with before treatment in the same group; ${ }^{b} P<0.05$ compared with the control group after treatment.

Table 6: Comparison of thoracic and lung compliance between two groups before and after treatment $\left(^{\bar{x} \bar{x}}{ }_{ \pm \mathrm{s}}\right)$

\begin{tabular}{llllc}
\hline Group & & Case number & Thoracic compliance & Lung compliance \\
\hline Observation group & Before treatment & 41 & $583.25 \pm 27.41$ & $320.93 \pm 17.42$ \\
& After treatment & 41 & $793.42 \pm 34.51^{\text {ab }}$ & $394.52 \pm 19.84^{\mathrm{ab}}$ \\
\multirow{2}{*}{ Control group } & Before treatment & 41 & $579.94 \pm 26.58$ & $318.76 \pm 16.58$ \\
& After treatment & 41 & $715.21 \pm 30.92^{\mathrm{a}}$ & $369.29 \pm 18.56^{\mathrm{a}}$ \\
\hline
\end{tabular}

Note: ${ }^{a}<<0.05$ compared with before treatment in the same group; ${ }^{b} P<0.05$ compared with the control group after treatment. 
Table 7: Comparison of CAT score between two groups before and after treatment $\left(\bar{X} \bar{X}_{ \pm \mathrm{S}}\right)$

\begin{tabular}{llll}
\hline Group & & Case number & CAT(point) \\
\hline Observation group & Before treatment & 41 & $28.42 \pm 3.15$ \\
& After treatment & 41 & $16.59 \pm 1.87^{\mathrm{ab}}$ \\
Control group & Before treatment & 41 & $28.15 \pm 2.97$ \\
& After treatment & 41 & $22.76 \pm 2.41^{\mathrm{a}}$ \\
\hline
\end{tabular}

Note: ${ }^{\mathrm{a}} P<0.05$ compared with before treatment in the same group; ${ }^{\mathrm{b}} P<0.05$ compared with the control group after treatment.

Table 8: Comparison of pulmonary functions between two groups before and after treatment $\left(\bar{x} \bar{x}_{ \pm s}\right)$

\begin{tabular}{lllll}
\hline Group & & Case number & FEV1\% (\%) & FEV1/FVC (\%) \\
\hline Observation group & Before treatment & 41 & $51.32 \pm 4.97$ & $54.28 \pm 3.91$ \\
& After treatment & 41 & $68.97 \pm 5.42^{\mathrm{ab}}$ & $70.16 \pm 5.04^{\mathrm{ab}}$ \\
\multirow{2}{*}{ Control group } & Before treatment & 41 & $50.28 \pm 6.35$ & $53.17 \pm 4.25$ \\
& After treatment & 41 & $62.13 \pm 3.21^{\mathrm{a}}$ & $63.50 \pm 2.87^{\mathrm{a}}$ \\
\hline
\end{tabular}

Note: ${ }^{\mathrm{a}}<<0.05$ compared with before treatment in the same group; ${ }^{\mathrm{b}} P<0.05$ compared with the control group after treatment.

\subsection{Comparison of complications between two groups}

Among 41 patients in the observation group, there was hyperglycaemia in one patient; among 41 patients in the control group, there were hyperglycaemia in 3 patients and upper gastrointestinal hemorrhage in one patient. The rates of complications were not statistically different between the two groups $(P>0.05)$.

\section{Discussion}

COPD mainly involves lungs and can also cause extrapulmonary manifestations such as malnutrition, anxiety, depression, and osteoporosis [14-16]. As a development of environmental pollution and population aging, the incidence rate, disability rate, and mortality rate of COPD are increased [17-19]. The repetitive acute exacerbation of COPD can cause continuous decrease of lung function mostly complicated with malnutrition, which causes poor immune function and respiratory failure $[6,20,21]$.

In the recent years, it has been proven that noninvasive mechanical ventilation has a good effect on patients with COPD complicated with respiratory failure. It is convenient, efficient, and good for observing the patient condition and supportive treatment, which significantly decreases the tracheal intubation rate, mortality, and length of stay in the hospital [22-24]. In noninvasive positive mechanical ventilation, the positive end-expiratory pressure and pressure support ventilation are combined to give pressure support during inspiration to decrease the load of respirator muscle and overcome the airway resistance; it is helpful to open the airway, promote the even distribution of air in the lung and oxygen diffusion, and avoid alveolar collapse. Furthermore, it can improve the air distribution and ventilation/perfusion ratio, which is helpful to the expiration of $\mathrm{CO}_{2}$ [25-27]. Aerosol inhalation of glucocorticoid is the main method of treating COPD complicated with respiratory failure at present, and budesonide is one of the preferred medicines [28,29]. Budesonide is a novel synthetic non-halogenate hormone with high lipophicity. Aerosol inhalation of budesonide can directly function on the disease site and rapidly improve the symptoms of COPD. It is reported that aerosol inhalation of budesonide can decrease airway resistance and pressure, effectively improve blood gas parameters, and shorten duration of mechanical ventilation and length of stay in hospital $[30,31]$. The results in this study showed that the total effective rate in the observation group was higher than the control group, IgA and IgG in the observation group were higher than the control group after treatment, CAT score in the observation group was lower than the control group after treatment, and FEV1\% and FEV1/ FVC in the observation group were higher than the control 
group. This suggests that budesonide combined with noninvasive mechanical ventilation can improve the curative effect, improve the symptoms, improve the thoracic and lung compliance of patients, improve the humoral immune function of patients, and improve the life quality of patients. The results in this study showed that serum PCT and STREM- 1 in the observation group were lower than the control group, suggesting that budesonide combined with noninvasive ventilation can decrease serum PCT and sTREM-1 level. PCT is a glycoprotein and the propeptide of calcitonin. PCT concentration is significantly increased in the early stage even when there are atypical clinical manifestations or immune suppression, and the increase degree is positively related to the infection severity $[32,33]$. STREM-1 is a member of the immune globulin superfamily related to inflammation, which is mainly expressed in myeloid cells such as macrophage, monocyte, and neutrophils. When there is infection with extracellular bacteria, STREM-1 expression is significantly up-regulated [34-36].

In conclusion, Budesonide combined with noninvasive mechanical ventilation has good curative effects in treating AECOPE patients complicated with type II respiratory failure. It can decrease serum PCT and STREM1 , increase thoracic lung compliance, and improve the humoral immune function and life quality.

Ethics approval and consent to participate: This study was approved by the Committee on Ethics of Biomedicine of Yulin First People's Hospital. This study also complied with the Declaration of Helsinki, and signed, written informed consent was obtained from all subjects included in this study.

Consent for publication: Not applicable.

Competing interests: The authors declare that they have no competing interests.

\section{Funding Sources: None.}

Authors' contributions: 1) Study conception and design: E.G.; 2) Sample acquisition and collection of clinical data: C.Z.; 3) Experiments conduct:E.G. ; 4) Data analysis: C.Z.; 5) Manuscript writing: E.G.;6) Editing and Reviewing: J.W.

Acknowledgement: Not applicable. Availability of data and materials: The datasets used and/or analyzed during the current study are available from the corresponding author on reasonable request.

\section{References}

[1] Cheng S.L., Chan M.C., Wang C.C., Lin C.H., Wang H.C., Hsu J.Y., et al. COPD in Taiwan: a National Epidemiology Survey. Int J Chron Obstruct Pulmon Dis, 2015, 10, 2459-2467; doi:10.2147/COPD.S89672

[2] Raluy-Callado M., Lambrelli D., MacLachlan S., Merinopoulou E., Hagan M.A., Khalid J.M. Epidemiology and Severity of Chronic Obstructive Pulmonary Disease (COPD) in the United Kingdom (UK). Value Health, 2014, 17, A590; doi:10.1016/j. jval.2014.08.2019

[3] Smith M.C., Wrobel J.P. Epidemiology and clinical impact of major comorbidities in patients with COPD. Int J Chron Obstruct Pulmon Dis, 2014, 9, 871-888; doi:10.2147/COPD. S49621

[4] Al Aqqad S.M., Tangiisuran B., Hyder Ali I.A., Kassim R.M., Lin W.J., Tengku Ismail T.S. Hospitalisation of multi-ethnic older patients with AECOPD: Exploration of the occurrence of anxiety, depression and factor associated with short term hospital readmission. Clin Respir J, 2016; doi:10.1111/ crj.12448

[5] Peng L., Ren P.W., Liu X.T., Zhang C., Zuo H.X., Kang D.Y., et al. Use of noninvasive ventilation at the pulmonary infection control window for acute respiratory failure in AECOPD patients: A systematic review and meta-analysis based on GRADE approach. Medicine (Baltimore), 2016, 95, e3880; doi:10.1097/MD.0000000000003880

[6] Song Y., Chen R., Zhan Q., Chen S., Luo Z., Ou J., et al. The optimum timing to wean invasive ventilation for patients with AECOPD or COPD with pulmonary infection. Int J Chron Obstruct Pulmon Dis, 2016, 11, 535-542; doi:10.2147/COPD. S96541

[7] Esquinas A.M., Confalonieri M. Anemia and health performance score evaluation as decisive factors for noninvasive mechanical ventilation decisions in AECOPD: are there new key cornerstones? Int J Chron Obstruct Pulmon Dis, 2014, 9, 151-152; doi:10.2147/COPD.S57085

[8] Yan H.Y., Yang Y., Wu Y.L. Clinical analysis of optimal timing for application of noninvasive positive pressure ventilation in treatment of AECOPD patients. Eur Rev Med Pharmacol Sci, 2014, 18, 2176-2181;

[9] Ding Z., Li X., Lu Y., Rong G., Yang R., Zhang R., et al. A randomized, controlled multicentric study of inhaled budesonide and intravenous methylprednisolone in the treatment on acute exacerbation of chronic obstructive pulmonary disease. Respir Med, 2016, 121, 39-47; doi:10.1016/j.rmed.2016.10.013

[10] Liapikou A., Torres A. Pharmacotherapy for lower respiratory tract infections. Expert Opin Pharmacother, 2014, 15, 2307-2318; doi:10.1517/14656566.2014.959927

[11] Segreti A., Fiori E., Calzetta L., Sabatini M., Segreti V., Rogliani P., et al. The effect of indacaterol during an acute exacerbation of COPD. Pulm Pharmacol Ther, 2013, 26, 630-634; doi:10.1016/j.pupt.2013.03.020

[12] Wells J.M., Morrison J.B., Bhatt S.P., Nath H., Dransfield M.T. Pulmonary Artery Enlargement Is Associated With Cardiac Injury During Severe Exacerbations of COPD. Chest, 2016, 149, 1197-1204; doi:10.1378/chest.15-1504 
[13] Mittal R., Chhabra S.K. GOLD Classification of COPD: Discordance in Criteria for Symptoms and Exacerbation Risk Assessment. COPD, 2017, 14, 1-6; doi:10.1080/15412555.201 6.1230844

[14] Liu Y., Liu Y.L., Cheng W., Yin X.M., Jiang B. The expression of SIRT3 in primary hepatocellular carcinoma and the mechanism of its tumor suppressing effects. Eur Rev Med Pharmacol Sci, 2017, 21, 978-998;

[15] Labarca G., Bustamante A., Valdivia G., Diaz R., Huete A., Mac Nab P., et al. The boundaries of mild chronic obstructive pulmonary disease (COPD): design of the searching clinical COPD onset (SOON) study. BMJ Open, 2017, 7, e015731; doi:10.1136/bmjopen-2016-015731

[16] Stephenson J.J., Wertz D., Gu T., Patel J., Dalal A.A. Clinical and economic burden of dyspnea and other COPD symptoms in a managed care setting. Int J Chron Obstruct Pulmon Dis, 2017, 12, 1947-1959; doi:10.2147/COPD.S134618

[17] Kim D.K., Hersh C.P., Washko G.R., Hokanson J.E., Lynch D.A., Newell J.D., et al. Epidemiology, radiology, and genetics of nicotine dependence in COPD. Respir Res, 2011, 12, 9; doi:10.1186/1465-9921-12-9

[18] Moretti A.M., Tafuri S., Parisi D., Germinario C. Epidemiology and costs of hospital care for COPD in Puglia. Multidiscip Respir Med, 2011, 6, 299-304; doi:10.1186/2049-69586-5-299

[19] Ozsancak Ugurlu A., Habesoglu M.A. Epidemiology of NIV for Acute Respiratory Failure in COPD Patients: Results from the International Surveys vs. the "Real World". COPD, 2017, 14, 429-438; doi:10.1080/15412555.2017.1336527

[20] Sun X., He Z., Zhang J., Deng J., Bai J., Li M., et al. Compare the efficacy of inhaled budesonide and systemic methylprednisolone on systemic inflammation of AECOPD. Pulm Pharmacol Ther, 2015, 31, 111-116; doi:10.1016/j. pupt.2014.09.004

[21] Wood J. The use of positive expiratory pressure therapy does not appear to be effective in people hospitalised with an acute exacerbation of chronic obstructive pulmonary disease (AECOPD). J Physiother, 2015, 61, 43; doi:10.1016/j. jphys.2014.11.009

[22] Dretzke J., Moore D., Dave C., Mukherjee R., Price M.J., Bayliss S., et al. The effect of domiciliary noninvasive ventilation on clinical outcomes in stable and recently hospitalized patients with COPD: a systematic review and meta-analysis. Int J Chron Obstruct Pulmon Dis, 2016, 11, 2269-2286; doi:10.2147/ COPD.S104238

[23] Petroianni A., Esquinas A.M. Noninvasive mechanical ventilation on the ward for severe COPD: still unresolved question of balance among safety and drawbacks? Int J Chron Obstruct Pulmon Dis, 2016, 11, 2209-2210; doi:10.2147/ COPD.S117861

[24] Yalcinsoy M., Salturk C., Oztas S., Gungor S., Ozmen I., Kabadayi F., et al. Can patients with moderate to severe acute respiratory failure from COPD be treated safely with noninvasive mechanical ventilation on the ward? Int J Chron Obstruct Pulmon Dis, 2016, 11, 1151-1160; doi:10.2147/COPD. S104801

[25] Braune S., Sieweke A., Brettner F., Staudinger T., Joannidis M., Verbrugge S., et al. The feasibility and safety of extracorporeal carbon dioxide removal to avoid intubation in patients with COPD unresponsive to noninvasive ventilation for acute hypercapnic respiratory failure (ECLAIR study): multicentre case-control study. Intensive Care Med, 2016, 42, 1437-1444; doi:10.1007/s00134-016-4452-y

[26] Duiverman M.L., Huberts A.S., van Eykern L.A., Bladder G., Wijkstra P.J. Respiratory muscle activity and patient-ventilator asynchrony during different settings of noninvasive ventilation in stable hypercapnic COPD: does high inspiratory pressure lead to respiratory muscle unloading? Int J Chron Obstruct Pulmon Dis, 2017, 12, 243-257; doi:10.2147/COPD. S119959

[27] Rialp G., Forteza C., Muniz D., Romero M. Role of First-Line Noninvasive Ventilation in Non-COPD Subjects With Pneumonia. Arch Bronconeumol, 2017, 53, 480-488; doi:10.1016/j.arbres.2016.08.017

[28] Haque R., Hakim A., Moodley T., Torrego A., Essilfie-Quaye S., Jazrawi E., et al. Inhaled long-acting beta2 agonists enhance glucocorticoid receptor nuclear translocation and efficacy in sputum macrophages in COPD. J Allergy Clin Immunol, 2013, 132, 1166-1173; doi:10.1016/j.jaci.2013.07.038

[29] Verhoeven G.T., Wijkhuijs A.J., Hooijkaas H., Hoogsteden H.C., Sluiter W. Effect of an inhaled glucocorticoid on reactive oxygen species production by bronchoalveolar lavage cells from smoking COPD patients. Mediators Inflamm, 2000, 9 , 109-113; doi:10.1080/096293500411578

[30] Gao S.Y., Huang J.Q., Luo Y.F., Li Z.P., Xie C.M., Guo Y.B. Comparison of the lung function change in patients with COPD and bronchial asthma before and after treatment with budesonide/formoterol. J Thorac Dis, 2012, 4, 583-587; doi:10.3978/j.issn.2072-1439.2012.11.02

[31] White P. Inhaled fluticasone and budesonide increased the risk of serious pneumonia in COPD. Evid Based Med, 2014, 19, 116; doi:10.1136/eb-2013-101695

[32] Picart J., Moiton M.P., Gauzere B.A., Gazaille V., Combes X., DiBernardo S. Introduction of a PCT-based algorithm to guide antibiotic prescription in COPD exacerbation. Med Mal Infect, 2016, 46, 429-435; doi:10.1016/j.medmal.2016.07.008

[33] Chang C.H., Tsao K.C., Hu H.C., Huang C.C., Kao K.C., Chen N.H., et al. Procalcitonin and $\mathrm{C}$-reactive protein cannot differentiate bacterial or viral infection in COPD exacerbation requiring emergency department visits. Int J Chron Obstruct Pulmon Dis, 2015, 10, 767-774; doi:10.2147/COPD.S76740

[34] Altay F.A., Elaldi N., Senturk G.C., Altin N., Gozel M.G., Albayrak Y., et al. Serum STREM-1 level is quite higher in Crimean Congo Hemorrhagic Fever, a viral infection. J Med Virol, 2016, 88, 1473-1478; doi:10.1002/jmv.24496

[35] Molad Y., Pokroy-Shapira E., Kaptzan T., Monselise A., Shalita-Chesner M., Monselise Y. Serum soluble triggering receptor on myeloid cells-1 (sTREM-1) is elevated in systemic lupus erythematosus but does not distinguish between lupus alone and concurrent infection. Inflammation, 2013, 36, 1519-1524; doi:10.1007/s10753-013-9694-z

[36] Su L., Liu D., Chai W., Liu D., Long Y. Role of sTREM-1 in predicting mortality of infection: a systematic review and meta-analysis. BMJ Open, 2016, 6, e010314; doi:10.1136/ bmjopen-2015-010314 